\title{
Pulmonary and Other Non-Neurological Vascular Malformations: Diagnosis and Endovascular Treatment
}

\author{
Yasutaka Baba, Sadao Hayashi and \\ Masayuki Nakajo \\ Additional information is available at the end of the chapter \\ http://dx.doi.org/10.5772/56730
}

\section{Introduction}

Since the International Society for the Study of Vascular Anomalies (ISSVA) was founded in 1992, the confused classification of vascular anomalies has improved and classified into 2 major categories; vascular tumors and vascular malformations and arteriovenous malformation (AVM) or arteriovenous fistula (AVF) belong to vascular malformations [1]. Although there has been a definite differentiation of pathological and clinical findings between vascular tumors and vascular malformations [1], new findings of vascular malformations have trickled in by some recent molecular biological study of vascular malformation [2-6]. There appears no clear difference in definition between AVM and AVF at this moment. Therefore we used AVM or AVF interchangeably according to the involved organs and previous papers.

We present our experiences in diagnosis and endovascular treatment of non-neurological vascular malformations. First of all, we address diagnosis and endovascular treatment of pulmonary AVMs. The symptoms of pulmonary AVMs include hypoxia, hemo-sputum, hyper-coagulated state, and paradoxical embolism. In addition, exciting new knowledge of pulmonary AVMs, including hereditary factors and cytokines such as vascular endothelial growth factor (VGEF) releasing phenomenon within the venous sac, are now highlighted. Endovascular treatment and long term follow-up results of pulmonary AVMs are also discussed. About vascular malformations involving other organs, we will present and illustrate a wide spectrum of vascular malformations involving neck, extremity, liver, common bile duct, pancreas, kidney, intestine, pelvic and uterine. 


\section{Chest}

\subsection{Diagnosis and endovascular treatment of Pulmonary AVMs (PAVMs)}

PAVMs are caused by abnormal communications between pulmonary arteries and pulmonary veins without the capillary beds and are most commonly congenital in nature. PAVMs are usually classified into the simple type with one feeding segmental artery and the complex type with 2 or more feeding segmental arteries. Definite diagnosis of PAVMs is made by contrast enhanced computed tomography and pulmonary angiography [7-9].

The right-to-left shunt causes the symptoms of PAVMs and results in hypoxia, dyspnea, cyanosis, and polycythemia when the shunt is developed. Besides this, lack of filter function due to absence of capillary beds induces the symptoms of paradoxical embolism and may results in brain abscess (10) or infarction. Although recently, small PAVMs are discovered on multi-detector row computed tomography (MDCT), the feeding pulmonary artery $3 \mathrm{~mm}$ or greater in diameter is generally indicated for treatment of PAVMs in order to block the nidus (sac) of PAVMs [9.11].

Embolotherapy has been the treatment option for PAVMs because it is minimally invasive, easy to perform and achieves a high success rate [7,8]. Microcoils have been used to treat PAVMs, but vascular plugs may be next generation devices to treat PAVMs [12]. However, incomplete occlusion of the sac or recanalization of an embolized feeding artery during a longterm follow-up could be seen in clinical setting. Remy-Jardin et al [7] reported that the overall treatment success rate was $75 \%$ at long term follow- ups (2-21 years) and repeated embolotherapy was required for recanalized PAVM and occurred in $19 \%$ patients. Meanwhile, Pollak et al [8] reported that problems related with embolized PAVMs occurred in $23 \%$ of treated patients at several interval follow-up points (over 3-7 years) and symptomatic events related to recanalization included respiratory symptoms, cerebral ischemia, brain abscess, hemoptysis and seizure. Like Remy-Jardin et al.[7], Pollak et al [8] emphasized that early recognition of the recanalized sac on follow-up imaging modality (computed tomography) and repeated embolotherapy are necessary to prevent recurrent symptoms related with PAVMs. In our experience, the overall treatment success rate was $70 \%$ at long term follow-up series (from 1989 to 2009) and the major cause of failed cases hinges on recanalized emolized arteries, in which repeated embolotherapy was required. However, symptoms related with recanalized PAVMs were not recognized in our series.

\subsection{Sac Embolization is adequate strategy for treatment of PAVMs}

We define here reperfusion as sac blood flow irrespective of the inflow route and recanalization as sac blood flow from the previously or successfully embolized arterial portion, respectively. Our colleagues [13] have reported a high rate (57\%) of reperfusion of PAVMs with steel coils and documented that the bronchial artery is one of the routes for reperfusion after embolotherapy for PAVMs. Therefore, we intend to embolize the sac itself with microcoils when the sac is $30 \mathrm{~mm}$ or less in diameter to prevent reperfusion in our institute. In the comparative study between the feeding artery embolization and sac embolization groups, the former 
showed a significant higher reperfusion rate at long term follow-ups (soon published). Reperfusion mainly depended on the type and number of used coils.

\subsection{The causes of reperfusion of embolized PAVMs (Fig. 1)}

Mechanisms underlying reperfusion of the embolized PAVMs were summarized by Pollak et al. [8] as: i) recanalization of the embolized vessel; ii) growth of a missed or previously small accessory artery; iii) bronchial and other systemic artery collateral flow into the pulmonary artery beyond the level of the embolization (creating a left-to-left shunt); and iv) pulmonary artery-to-pulmonary artery collateral flow about the occlusion. Reperfusion may occur individually or in combination of the aforementioned mechanisms. Mechanism iv, has been observed only in young children and presumably results from the ability for continued lung growth [8].

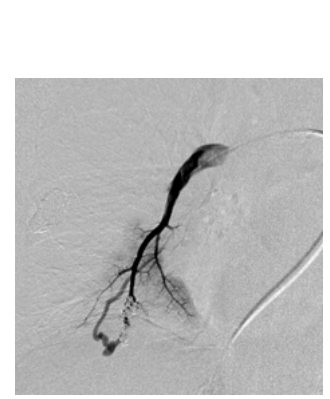

(a)

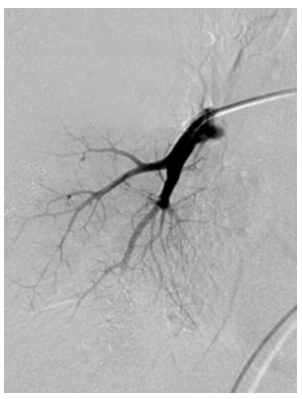

(b)

Figure 1. Pulmonary AVM. (a) Right middle branch of pulmonary arteriogram shows recanalized nidus through the previous embolized feeding vessel with microcoils. (b) Right middle branch of pulmonary arteriogram shows complete embolized nidus after additional coil embolization.

\subsection{Some cytokines are released via a sac?}

Dupuis-Girod et al [6] have reported that bevacizumab has an effect on reducing the symptoms relating with high cardiac output or PAVMs regression in patients with PAVMs in hereditary hemorrhagic telangiectasia (HHT) that exhibits AVMs in several different organs. Some cytokines like VGEF and TGF beta may have relationship with development of pulmonary AVMs [2-6] and anti VGEF agents have a potential to regression of PAVMs [2]. However, Giordano et al mentioned that VGEF and TGF beta are not useful for diagnosing HHT [3].

\subsection{Systemic circulation to thoracic AVMs (Fig.2)}

Another specific type of thoracic AVMs is mediastinal AVM, which is difficult to manage the symptoms relating with high flow shunting, for example, hemoptysis and hemothorax [14]. Unlike PAVMs, the shunt blood flow is derived from systemic arterial blood flow and untreated various systemic arterial branches could develop even if embolotherapy is attempt- 
ed. Therefore it is very hard to block the arterial blood inflow to the nidus, resulting in recurrent symptoms related with thoracic AVMs.

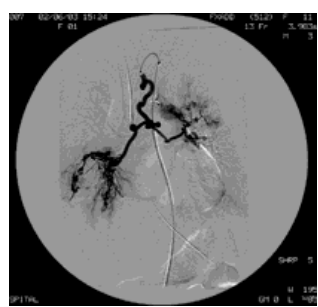

(a)

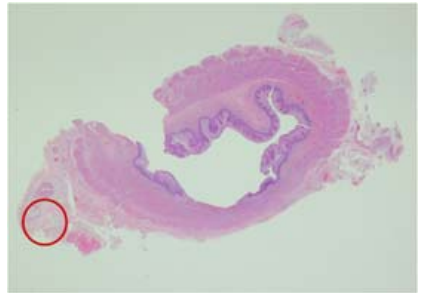

(c)

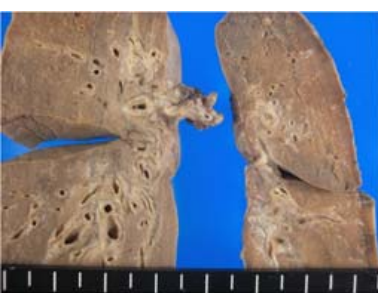

(b)

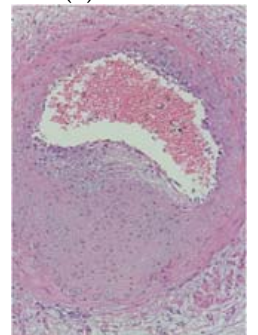

(d)

Figure 2. Mediastinal AVMs.(a) Bronchial arteriogram shows neovascularization and patchy staining at bilateral lung hilum. There are many systemic arterial supplies to these mediastinal lesions (not shown). (b) Gross speciemen of the resected lungs show the hypertrophied bronchial walls and the hilums. (c) Microscopic speciemen of the resected esophagus shows neovascularization around the esophagus (red circle). (d) Microscopic speciemen in which red circle denotes reveals the proliferation of the vascular wall.

\section{Other non-neurological AVFs}

\subsection{Neck AVMs (Fig.3)}

Unlike other peripheral vascular AVMs, it is difficult to manage neck AVMs and control the symptoms. Particularly embolotherapy is difficult to perform because of high risk of cerebrovascular events after treatment and fortified vascular supply to the nidus, resulting in recurrent AVMs. Ordinal embolotherapy with gelatin, coils, and n-butyl cyanoacrylate (NBCA) could not become a breakthrough treatment option for head and neck AVMs. A recent clinical study showed that ethanol sclerotherapy is promising for treating head and neck AVMs [15]. Ethanol is a toxic substance that destroys the endothelial wall permanently and results in thrombus formation [16]. However, it needs to pay attention to blood flow speeds via the nidus because absolute ethanol has a risk of lethal direct tissue injury including pulmonary artery collapse [16]. Therefore, remission of blood flow via the nidus by an appropriate method (for instance, using balloon catheter, coils, or other liquid agents) is essential to exploit absolute ethanol. 


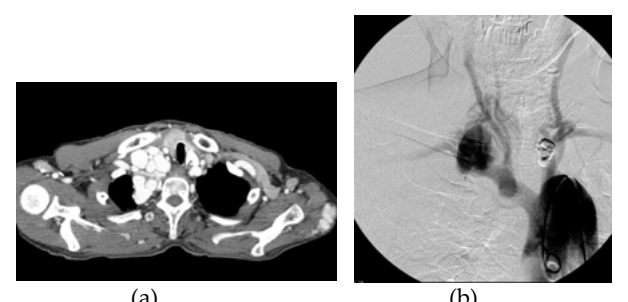

(a)

(b)

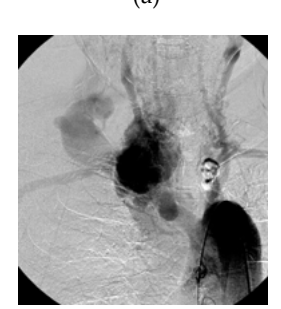

(c)

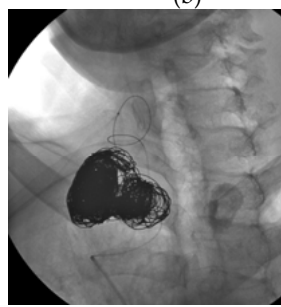

(d)

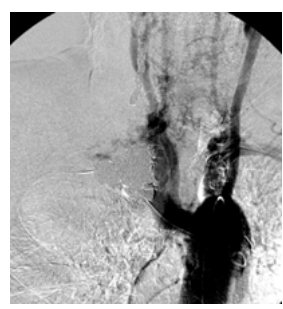

(e)

Figure 3. Neck AVMs. (a) Contrast enhanced computed tomography (CT) shows that torturous and dilated vessels at the right neck. (b) Aortogram shows neovascularization and dilated vessels around the right neck. (c) Aortogram also shows that early visualization of the dilated and torturous veins around the right neck. (d) Coil embolization via both arterial and venous sides was performed repeatedly. (e) After coil embolization, despite of incomplete embolization, aortogram shows the diminish blood flow via the nidus.

\subsection{Liver AVFs: HCCs/ HHT (Fig.4)}

In patients with HHT, liver is frequently involved and they show a wide spectrum of clinical manifestations. In patients with HHT, traces of elements that pass through hepatic AVMs, could affect the extrapyramidal movement via depositing manganese in the globus pallidus [17]. On the contrary, hepatocellular carcinomas (HCC) easily invades hepatic venous structures according to its progression, result in hepatic arteriovenous fistulas. In patients with AV fistulas due to HCC, it is difficult to treat them by embolotherapy because fistula channels are derived from tumor vasculatures. We experienced a case of HCC whose AV fistula was successfully treated by absolute ethanol [18].

\subsection{Common bile ducts AVFs: Hereditary Hemorrhagic Telangiectasia (HHT) (Fig.5)}

HHT involves rarely the common bile duct. Hemobilia may occur because AVMs may protrude from the common bile duct wall. We experienced a patient with HHT who had a small AVM of the common bile duct causing intractable hemobilia [19].

Later, intractable hemobilia ceased by superselective coil embolization (Fig.5) and have not recurred. Other clinical findings associated with HHT, for instance, intractable epistaxis, telangiectasis of the lip, tongue, and oral cavity might be key to reach the etiology of hemobilia. Otherwise, it is difficult to recognize the imaging findings associated with HHT except selective angiography. 


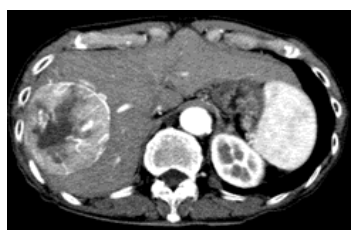

(a)

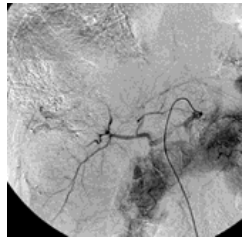

(d)

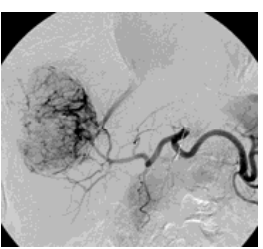

(b)

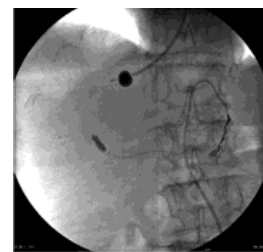

(c)

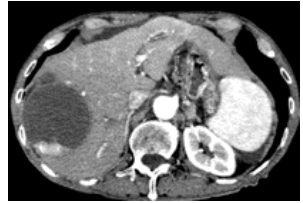

(e)

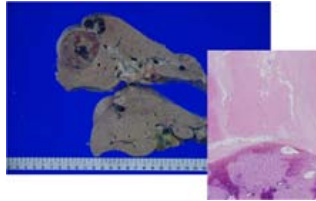

(f)

Figure 4. Hepatocellular carcinoma with arteriovenous shunting. (All figures are cited under permission from publishing company: reference 18) (a) Contrast enhanced CT shows the hypervascular tumor in the right liver lobe. (b) Celiac arteriogram shows not only the hypervascular liver tumor but also early visualization of the right hepatic vein. (c) We attempted to embolize intratumoral shunting by way of combined balloon inflation via both hepatic arterial and venous sides. Under this condition, the intratumoral shunting can be embolized with absolute etanol. (d) After embolization with absolute ethanol, celiac arteriogram shows vanishing of intratumoral shunting and hypervascular liver tumor. (e) After these procedures, on contrast enhanced CT, the vast majority of hypervascualr tumor shows necrosis. (f) On macroscopic (left) and microscopic (right) specimens of the resected liver, the major part of the liver tumor shows necrosis. Reproduced from reference [18] with the permission of the publisher.

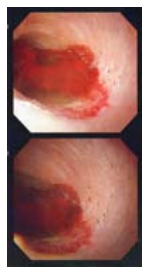

(a)

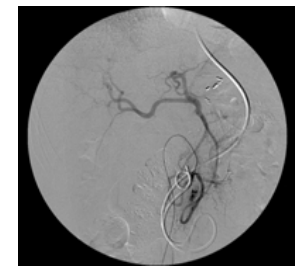

(b)

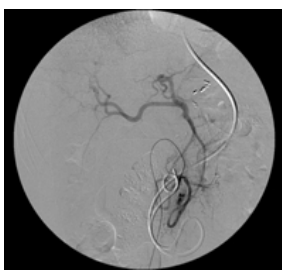

(c)

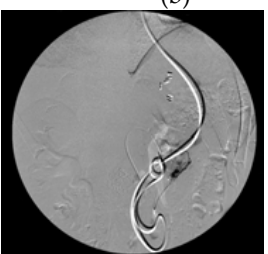

(d)

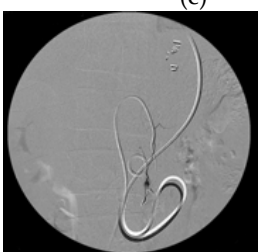

(e)

Figure 5. AVMs of common bile duct in a patient with hereditary hemorrhagic telangiectasis. (All figures are cited under permission from publishing company: reference 19) (a) Bile duct endoscopy shows bleeding in the bile duct lumen. (b) Inferior pancreaticoduodenal arteriogram shows patchy stain in the lower bile duct. (c) Selective arteriogram via the branch of pancreaticoduodenal arcade shows the staining in the lower bile duct. (d) After coil embolization of bleeding sites, the staining in the lower bile duct disappeared on amgiogram. Reproduced from reference [19] with the permission of the publisher. 


\subsection{Pancreatic AVFs: Post transplantation and Metastasis from Renal Cell Carcinoma (RCC)}

Apart from the case with HHT, postoperative pancreatic transplantation is the major cause of pancreas AVFs [20]. Another cause of pancreatic AVFs is metastatic pancreatic cancer from renal cell carcinoma. In both entities of pancreatic AVF, it could be difficult to manage the portal hypertension symptoms associated with inflowing systemic blood pressure to the portal system. Barth et al. [20] reported that elegant embolization via both transarterial and transvenous accesses for the pancreatic AVF case.

\subsection{Intestinal AVMs}

In the patients with obscure origin of gastrointestinal bleeding (GIB), small intestinal AVM is one of the sources that cause intractable GIB. Coil embolization of angiodysplasia have been reported, however, embolothearpy may not always successful to cease GIB and surgical intervention sometimes may be necessary [21-23].

When operation is performed in a patient with intestinal AVMs, placing coils might be help to recognize the location of intestinal AVMs.

\subsection{Colonic AVMs (Fig.6)}

Colonic AVM is rare and could induce portal hypertension and coil embolization may be feasible to treat it [24]. We experience a patient with rectal AVFs whose major part was thrombosed and calcified, but intractable rectal bleeding continued. Curative operation was performed later, the sysmptom associated with rectal AVF was resolved.

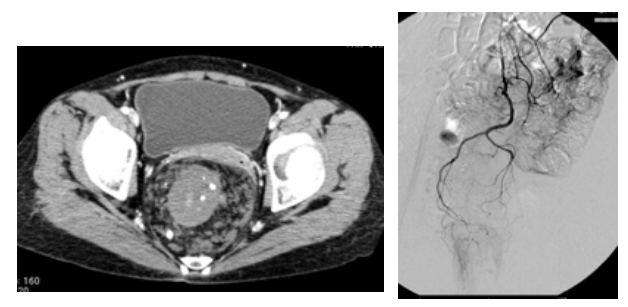

(a)

(b)

Figure 6. Rectal AVMs. (a) Contrast enhanced computed tomography (CT) shows no enhanced, thickening and calcified mass of the rectum, which was surrounded by pararectal venous varices. (b) Superior rectal arteriogram shows minor tumor staining without early venous drainage. Therefore, thrombotic change was suspected in the major part of arteriovenous malformation.

\subsection{Renal AVMs/AVFs (Fig.7,8)}

Renal AVF is a frequent disease but may be misdiagnosed unless classical findings of renal AVFs are depicted on ultrasonography (US) or contrast enhanced CT. We misdiagnosed renal AVF in a patient in whom contrast CT depicted aneurysmal dilatation at the level of renal 
hilum [25]. In renal AVFs/AVMs, embolotherapy could be a major option to treat and occlude the fistula or nidus.

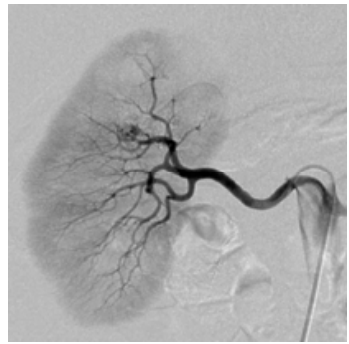

(a)

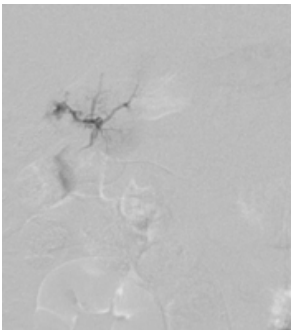

(b)

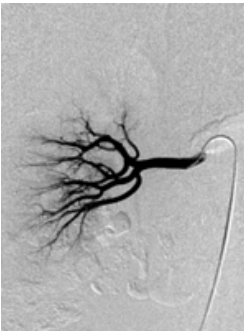

(c)

Figure 7. Renal AVM; (a) Right renal arteriogram shows staining of the upper pole of the kidney.(b) Early venous drainage is evident on super-selective arteriogram of the upper pole of the renal artery. (c)After embolization of the renal AVM with absolute ethanol, it disappeared.

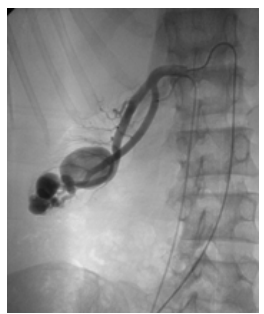

(a)

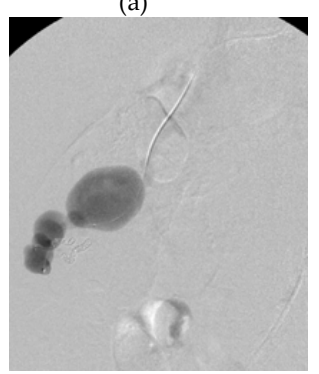

(d)

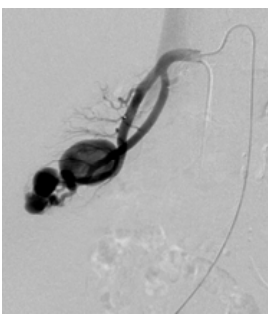

(b)

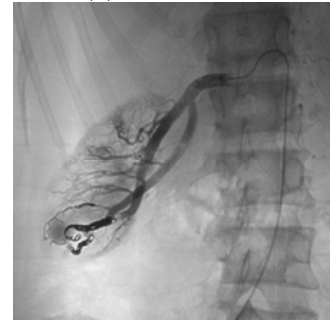

(e)

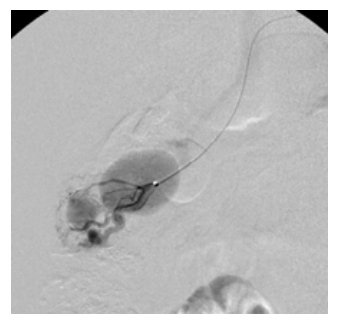

(c)

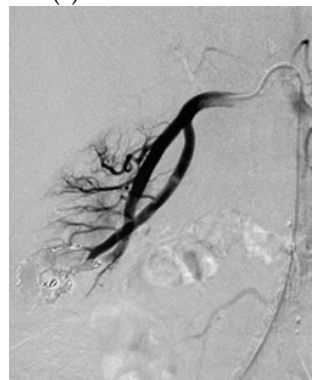

(f)

Figure 8. Renal AVF with venous aneurismal dilatation.(a)(b) Right renal arteriogram shows the hypertrophied renal artery and aneurismal dilatation of the right renal artery. (c) Super-selective renal arteriography discriminates between renal artery and aneurismal dilatation. (d) Renal venogram shows the direct communication between renal vein and anueurysmal dilatation. Therefore, aneurismal dilatation of the renal vein could be induced by arterialization of the renal vein due to arteriovenous fistula. (e), (f) After coil embolization of renal arteriovenous fistula with microcoils, the renal AVF disappeared. 


\subsection{Pelvic AVFs (Fig.9)}

Pelvic AVF is rare. It is also difficult to manage or control it. Because it may have numerous and complex vessels, embolotherapy via the arterial side has a limitation to occlude the nidus. Do et al. [26] reported high complete regression rate (83.3\%) by arterial ethanol embolotherapy combined with venous coil embolization. A case was reported whose pelvic AVF was successfully treated by venous embolization under balloon occlusion [27]. This technique is usually used for treatment of gastric varices and portal hypertension [28].

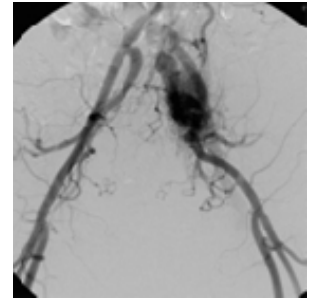

(a)

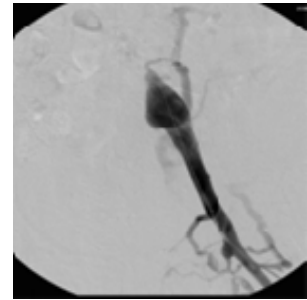

(b)

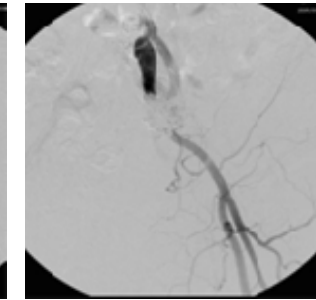

(c)

Figure 9. Pelvic AVMs. (a) Aortography shows fine vessels proliferation around the left iliac artery and subsequent early visualization of left iliac veins. (b)Venography shows occlusion of the left common iliac vein. (c)Fine vessels proliferation and early venous drainage are not observed by left common iliac arteriography.

\subsection{Uterine AVMs (Fig.10)}

The etiology of uterine AVFs covers a wide spectrum including iatrogenic and post dilate and curettage ones, vascular tumor, and trophoblastic disease [29]. Like other organ involved AVMs, controlling the blood flow of the nidus is difficult and surgical intervention (hysterectomy) is dangerous because intraoperative bleeding is tremendous [30]. Selective arterial embolotherapy is useful in some uterine AVMs cases, but we experienced an unsuccessful case of uterine AVMs that substituted for operative uterine artery clamping [31].

\subsection{Upper and lower extremity AVMs}

AVMs of the upper and lower extremities may manifest cosmetic distortion and painful tumor. Besides them, venous hypertension due to peripheral AVMs leads to bony osteolysis and distortion, sometimes results in pathological fracture. Do et al [32] have reported that patients with peripheral subcutaneous AVMs have the bone involvement in 59\% of the patients. For the management of residual subcutaneous AVMs, the authors have recommended that patients wear compression stockings rather than undergo additional ethanol embolotherapy, which can result in skin necrosis [32]. In addition, as mentioned previously [16], ablation of the AVMs require stasis of absolute ethanol within not only the nidus but also angiopathic feeding or drainage vessels. 


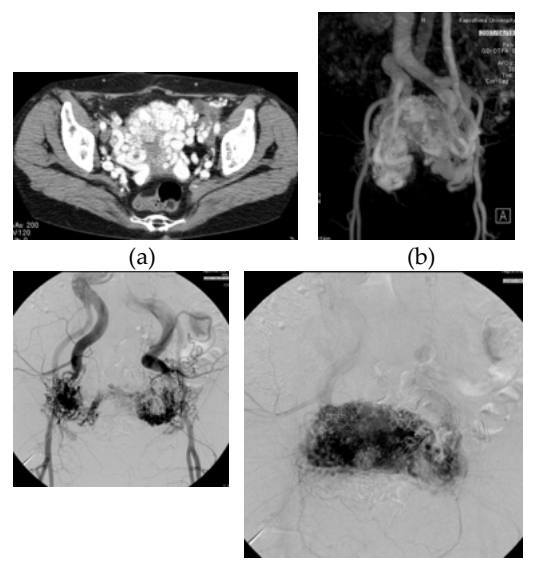

(c)

(d)

Figure 10. Uterine AVMs. Tortuous and dilated vessels are present in the entire uterus on contrast enhanced CT (a) and magnetic resonance arteriography (b). Pelvic arteriograms (c, d) show numerous and tortuous dilated vessels throughout the uterus ad early venous drainage. Reproduced from reference [31] with the permission of the publisher.

\subsection{Lower extremity AVFs with DVT (Deep Vein Thrombosis) (Fig.11)}

Venous obstruction mainly due to venous thrombosis is an underlying cause of phleboid disease [33-35]. However, reports of AVF in the leg are rare, and no guidelines have been developed for the treatment of AVFs in the leg [36,37]. Deep-vein thrombosis may be followed by Inflammation and neovascularity to form AVFs. First, low oxygenation due to thrombogenesis and various vascular growth factors increase the venous pressure. Chemical factors are then released by the vascular endothelium, vasa vasorum, and platelets to initiate the inflammatory process. Next, neovascularization induces thrombotic reconstitution and activates various inflammatory cells [37]. We therefore recommend restoring the venous occlusion before performing arterial embolization in patients with both AVF and venous occlusion.

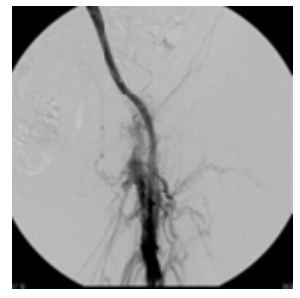

(a)

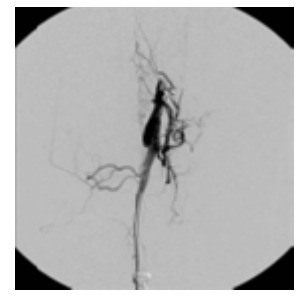

(b)

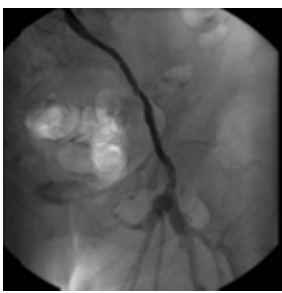

(c)

Figure 11. Leg AVFs with deep vein thrombosis. (a) Aortography shows fine vessels proliferation around the left internal iliac artery and early visualization of the left iliac vein. (b)Venography shows occlusion of the left common iliac vein. (c)After balloon PTA and stent deployment, venography shows recanalization of the left common iliac vein. 


\section{Conclusion}

Endovascular therapy including embolotherapy, sclerotherapy or their combination could be an option for treating a patient with AVM ( AVF), because complete resectability of AVMs is low. According to the location of AVMs, flow speed of the nidus and relation with other organs, appropriate embolic and sclerotic materials should be chosen to obtain good clinical outcome.

\section{Author details}

Yasutaka Baba*, Sadao Hayashi and Masayuki Nakajo

*Address all correspondence to: yasutaka@m3.kufm.kagoshima-u.ac.jp

Department of Radiology, Kagoshima University, Sakuragaoka, Kagoshima-shi, Kagoshima, Japan

\section{References}

[1] Odile Enjolras MWaRC. Introduction: ISSVA Classification. London: Cambridge University Press; 2007,pp 2-11.

[2] Lacout A, Marcy PY, Thariat J, El Hajjam M, Lacombe P. VEGF target in HHT lung patients: the role of bevacizumab as a possible alternative to embolization. Med Hypotheses. 2012; May;78(5) 689-90.

[3] Giordano P, Lenato GM, Pierucci P, Suppressa P, Altomare M, Del Vecchio G, et al. Effects of VEGF on phenotypic severity in children with hereditary hemorrhagic telangiectasia. J Pediatr Hematol Oncol. 2009; Aug;31(8) 577-582.

[4] Sabba C, Pasculli G, Lenato GM, Suppressa P, Lastella P, Memeo M, et al. Hereditary hemorrhagic telangiectasia: clinical features in ENG and ALK1 mutation carriers. J Thromb Haemost. 2007; Jun;5(6) 1149-1157.

[5] McDonald J, Pyeritz RE. Hereditary Hemorrhagic Telangiectasia. In: Pagon RA, Bird TD, Dolan CR, Stephens K, Adam MP. (ed.) Gene Reviews ${ }^{\text {TM }}$ [Internet]. Seattle (WA): University of Washington, Seattle; 1993-.2000 Jun 26 [updated 2012 Jan 05] http://www.ncbi.nlm.nih.gov/books/NBK1351/

[6] Dupuis-Girod S, Ginon I, Saurin JC, Marion D, Guillot E, Decullier E, et al. Bevacizumab in patients with hereditary hemorrhagic telangiectasia and severe hepatic vascular malformations and high cardiac output. JAMA. 2012; Mar 7;307(9) 948-955.

[7] Remy-Jardin M, Dumont P, Brillet PY, Dupuis P, Duhamel A, Remy J. Pulmonary arteriovenous malformations treated with embolotherapy: helical CT evaluation of 
long-term effectiveness after 2-21-year follow-up. Radiology. 2006; May;239(2) 576-585.

[8] Pollak JS, Saluja S, Thabet A, Henderson KJ, Denbow N, White RI, Jr. Clinical and anatomic outcomes after embolotherapy of pulmonary arteriovenous malformations. J Vasc Interv Radiol. 2006; Jan;17(1) 35-44.

[9] White RI, Jr., Lynch-Nyhan A, Terry P, Buescher PC, Farmlett EJ, Charnas L, et al. Pulmonary arteriovenous malformations: techniques and long-term outcome of embolotherapy. Radiology. 1988; Dec;169(3) 663-669.

[10] Mathis S, Dupuis-Girod S, Plauchu H, Giroud M, Barroso B, Ly KH, et al. Cerebral abscesses in hereditary haemorrhagic telangiectasia: a clinical and microbiological evaluation. Clin Neurol Neurosurg. 2011; Apr;114(3) 235-240.

[11] Lee DW, White RI, Jr., Egglin TK, Pollak JS, Fayad PB, Wirth JA, et al. Embolotherapy of large pulmonary arteriovenous malformations: long-term results. Ann Thorac Surg. 1997; Oct;64(4) 930-939; discussion 9-40.

[12] Cil B, Canyigit M, Ozkan OS, Pamuk GA, Dogan R. Bilateral multiple pulmonary arteriovenous malformations: endovascular treatment with the Amplatzer Vascular Plug. J Vasc Interv Radiol. 2006; Jan;17(1) 141-145.

[13] Sagara K, Miyazono N, Inoue H, Ueno K, Nishida H, Nakajo M. Recanalization after coil embolotherapy of pulmonary arteriovenous malformations: study of long-term outcome and mechanism for recanalization. AJR Am J Roentgenol. 1998; Mar;170(3) 727-730.

[14] Grillo HC, Athanasoulis CA. Tracheal obstruction from mediastinal arteriovenous malformation. J Thorac Cardiovasc Surg. 2004; Nov;128(5) 780-782.

[15] Pekkola J, Lappalainen K, Vuola P, Klockars T, Salminen P, Pitkaranta A. Head and Neck Arteriovenous Malformations: Results of Ethanol Sclerotherapy. AJNR Am J Neuroradiol. 2012; Jul 5. [Epub ahead of print]

[16] Baba Y, Miyazono N, Kanetsuki I, Nishi H, Hamada H, Nakajo M. Re: Combined arteriovenous malformation and aneurysm of the ulnar artery: successful arterial embolization by using absolute ethanol. Cardiovasc Intervent Radiol. 1999; May-Jun; 22(3) 266-267.

[17] Baba Y, Ohkubo K, Hamada K, Hokotate H, Nakajo M. Hyperintense basal ganglia lesions on T1-weighted images in hereditary hemorrhagic telangiectasia with hepatic involvement. J Comput Assist Tomogr. 1998; Nov-Dec;22(6) 976-979.

[18] Senokuchi T, Baba Y, Hayashi S, Nakajo M. Embolization of hepatic arteriovenous shunt with absolute ethanol in a patient with hepatocellular carcinoma. Cardiovasc Intervent Radiol.2010; Feb;34 Suppl 2 S154-156. 
[19] Hayashi S, Baba Y, Ueno K, Nakajo M. Small arteriovenous malformation of the common bile duct causing hemobilia in a patient with hereditary hemorrhagic telangiectasia. Cardiovasc Intervent Radiol. 2008; Jul 31 Suppl 2 S131-134.

[20] Barth MM, Khwaja K, Faintuch S, Rabkin D. Transarterial and transvenous embolotherapy of arteriovenous fistulas in the transplanted pancreas. J Vasc Interv Radiol. 2008; Aug;19(8) 1231-1235.

[21] Defreyne L, Verstraeten V, De Potter C, Pattyn P, De Vos M, Kunnen M. Jejunal arteriovenous malformation, diagnosed by angiography and treated by embolization and catheter-guided surgery: case report and review of literature. Abdom Imaging. 1998; Mar-Apr;23(2) 127-131.

[22] Gordhan AD, Newey CR, Wong G, Wieland J. N-butyl cyanoacrylate embolization of small bowel arteriovenous malformation presenting with acute massive lower gastrointestinal hemorrhage. J Vasc Interv Radiol. 2008; Nov;19(11) 1669-1670.

[23] Liao Z, Gao R, Li ZS. Vascular malformation of the small intestine. Endoscopy. 2007; Feb;39 Suppl 1:E319.

[24] Uthoff H, Pena C, Contreras F, Katzen BT. Symptomatic ascites caused by a longstanding posttraumatic mesenteric arteriovenous fistula. J Vasc Interv Radiol.2012; May; 23(5) 722-724.

[25] Cura M, Elmerhi F, Suri R, Bugnone A, Dalsaso T. Vascular malformations and arteriovenous fistulas of the kidney. Acta Radiol. 2010; Mar;51(2) 144-149.

[26] Do YS, Kim YW, Park KB, Kim DI, Park HS, Cho SK, et al. Endovascular treatment combined with emboloscleorotherapy for pelvic arteriovenous malformations. J Vasc Surg. 2011; Feb;55(2) 465-471.

[27] Mitsuzaki K, Yamashita Y, Utsunomiya D, Sumi S, Ogata I, Takahashi M, et al. Balloon-occluded retrograde transvenous embolization of a pelvic arteriovenous malformation. Cardiovasc Intervent Radiol. 1999; Nov-Dec;22(6) 518-520.

[28] Kanekawa H, Kayama A, Goto K, Kawanishi T, Yamazaki T, Mima S. [Balloon-occluded retrograde transvenous obliteration for treatment of gastroesophageal varices]. Nihon Geka Gakkai Zasshi. 1996; Jan;97(1) 78-82.

[29] Kwon JH, Kim GS. Obstetric iatrogenic arterial injuries of the uterus: diagnosis with US and treatment with transcatheter arterial embolization. Radiographics. 2002; JanFeb;22(1) 35-46.

[30] Cura M, Martinez N, Cura A, Dalsaso TJ, Elmerhi F. Arteriovenous malformations of the uterus. Acta Radiol. 2009; Sep;50(7) 823-829.

[31] Yokomine D, Yoshinaga M, Baba Y, Matsuo T, Iguro Y, Nakajo M, et al. Successful management of uterine arteriovenous malformation by ligation of feeding artery af- 
ter unsuccessful uterine artery embolization. J Obstet Gynaecol Res. 2009; Feb;35(1) 183-188.

[32] Do YS, Park KB, Park HS, Cho SK, Shin SW, Moon JW, et al. Extremity arteriovenous malformations involving the bone: therapeutic outcomes of ethanol embolotherapy. J Vasc Interv Radiol. 2010; Jun;21(6) 807-816.

[33] Fournier D, Rodesch G, Terbrugge K, Flodmark O, Lasjaunias P. Acquired mural (dural) arteriovenous shunts of the vein of Galen. Report of 4 cases. Neuroradiology. 1991; 33(1) 52-55.

[34] Ozawa T, Miyasaka Y, Tanaka R, Kurata A, Fujii K. Dural-pial arteriovenous malformation after sinus thrombosis. Stroke. 1998; Aug;29(8) 1721-1724.

[35] Phatouros CC, Halbach VV, Dowd CF, Lempert TE, Malek AM, Meyers PM, et al. Acquired pial arteriovenous fistula following cerebral vein thrombosis. Stroke. 1999; Nov;30(11) 2487-2490.

[36] Link DP, Garza AS, Monsky W. Acquired peripheral arteriovenous malformations in patients with venous thrombosis: report of two cases. J Vasc Interv Radiol. 2010; Mar; 21(3) 387-391.

[37] Labropoulos N, Bhatti AF, Amaral S, Leon L, Borge M, Rodriguez H, et al. Neovascularization in acute venous thrombosis. J Vasc Surg. 2005; Sep;42(3) 515-518. 